\title{
Spontaneous pneumomediastinum in COVID-19 pneumonia
}

\author{
Marco Loffi ${ }^{1}$, Valentina Regazzoni ${ }^{1}$, Pietro Sergio ${ }^{2}$, Enrico Martinelli ${ }^{3}$, Italo Stifani ${ }^{3}$, Filippo Quinzani ${ }^{1}$, \\ Debora Robba ${ }^{1}$, Annunziata Cotugno ${ }^{4}$, Meghi Dede ${ }^{5}$, Gian Battista Danzi ${ }^{1}$ \\ ${ }^{1}$ Division of Cardiology; ${ }^{2}$ Division of Radiology; ${ }^{3}$ Division of Pneumology, Cremona Hospital; ${ }^{4}$ Institute of Cardiology, \\ Department of Medical and Surgical Specialties, Radiological Sciences, and Public Health, University of Brescia; \\ ${ }^{5}$ University of Milan, Italy
}

\begin{abstract}
Spontaneous pneumomediastinum is a benign entity but can worsen the underlying condition with which it is associated. We evaluated the incidence and the clinical relevance of spontaneous pneumomediastinum in a consecutive series of 102 patients with
\end{abstract}

Correspondence: Valentina Regazzoni, Division of Cardiology, Ospedale di Cremona, Viale Concordia 1, 26100 Cremona, Italy. Tel. +39.0372.405322. E-mail: valentina15686@hotmail.it

Key words: Pneumomediastinum; pneumonia; COVID-19.

Contributions: All the co-authors, made substantial contribution to the realization of this work. In details, GBD conceived this study and supervised all aspects of its implementation. ML and VR collaborated in the inception of the study and carried out the analysis of the data. PS, EM, IS, AC, FQ, DR and MD collected the data and collaborated in the analysis. All the authors contributed to the interpretation of the results and the proof reading of the manuscript. All the co-authors approve the final version of the manuscript to be submitted and published.

Conflict of interest: The authors declare no conflict of interest.

Ethics approval and content to participate: The study was approved by the Ethical Committee of Cremona Hospital. Written informed consent was obtained from all patients included in this study.

Availability of data and materials: The data used to support the findings of this study are available from the corresponding author upon request.

Acknowledgments: The authors would like to thank all of the doctors, nurses and medical workers from Cremona Hospital for their efforts to combat the COVID-19 outbreak.

Received for publication: 22 May 2020.

Accepted for publication: 7 September 2020.

${ }^{\circ}$ Copyright: the Author(s), 2020

Licensee PAGEPress, Italy

Monaldi Archives for Chest Disease 2020; 90:1399

doi: 10.4081/monaldi.2020.1399

This article is distributed under the terms of the Creative Commons Attribution Noncommercial License (by-nc 4.0) which permits any noncommercial use, distribution, and reproduction in any medium, provided the original author(s) and source are credited.
COVID-19 pneumonia. Six cases of pneumomediastinum were identified by high-resolution chest CT-scan. Three patients required early intubation, and one of them died, while in in the remaining subjects the clinical course was benign. The presence of pneumomediastinum required some changes in the management of mechanical ventilation. In conclusion, spontaneous pneumomediastinum is a possible complication of severe COVID-19 pneumonia that can affect patient management and clinical outcomes.

\section{Introduction}

Spontaneous pneumomediastinum (SP) is rarely diagnosed in the general population, but frequently described during bacterial and viral infection [1,2]. It usually is a benign entity but can worsen the severity of the underlying condition. Northern Italy was the first European area that faced the Coronavirus Disease 2019 (COVID-19) and our hospital located in Cremona, Lombardy Region, was early converted in a COVID-19 dedicated center [3].

This is the first study that evaluate the incidence and the clinical relevance of SP in a consecutive series of patients with severe COVID-19 pneumonia.

\section{Materials and Methods}

We retrospectively analyzed clinical data from a sample of 187 patients admitted in our center with diagnosis of COVID-19 pneumonia between February $21^{\text {st }}$ to March $1^{\text {st }}, 2020$ using a computerized data collection system. The study was approved by our Ethical Committee.

Patients were assessed for eligibility on the basis of three principal inclusion criteria: i) a confirmed clinical and radiological diagnosis of COVID-19 pneumonia [4]; ii) a positive ReverseTranscriptase-Polymerase-Chain-Reaction assay for SARS-CoV2 in a respiratory tract sample; iii) a high-resolution chest computed tomography scan (HRCT) within 96 hours from admission.

We identified 102 patients who represent the study population. Relevant clinical and laboratory data were obtained from medical records. CT image analysis was performed by an experienced thoracic radiologist (P.S.). Acute Respiratory Distress Syndrome (ARDS) was defined and classified based on the ratio between $\mathrm{PaO} 2$ and $\mathrm{FiO} 2$ ratio according to WHO criteria [5].

Pneumonia severity was classified based on the lung involvement as mild, moderate and severe as reported in Table 1. The presence and characteristics of pneumomediastinum were described qualitatively. 


\section{Statistical analysis}

Continuous variables were expressed as median with its interquartile range (IQR) and compared with the Mann-Whitney U test. Categorical variables were described as number (\%), and proportions for categorical variables were compared using the Fisher exact test. A two-sided $\alpha$ of less than 0.05 was considered statistically significant. Statistical analyses were done using the SPSS version 21.0 software (IBM, New York, USA).

\section{Results}

Clinical characteristics, radiological data and the in-hospital outcomes of the 102 patients are reported in Table 1. Laboratory data are listed in Table 2. ARDS was present in $50 \%$ of patients at admission. SP was documented in six subjects $(6 \%)$. In three cases there was a large amount of air $(>20 \mathrm{~mm})$ in the mediastinum surrounding all of the vascular structures, infiltrating the neck and with evidence of subcutaneous emphysema (Figure 1A). In the remaining cases the presence of air was more limited (Figure 1B). The same three patients with large SP developed severe ARDS required intubation. In two of them the accumulation of air in the mediastinum increased significantly during mechanical ventilation despite the measures taken to reduce volutrauma. In one of these patients we had to escalate the degree of support because of the presence of pneumopericardium and extensive subcutaneous emphysema (Figure 1 C,D). This same patient died during hospitalization. The remaining five patients were discharge few weeks later with documented disappearance of pneumomediastinum at control CT-scan.

\section{Discussion}

The precise mechanism underlying the development of SP during respiratory infections is unknown $[1,2]$. One possible explanation is that severe pneumonia can cause diffuse alveolar damage and rupture, leading to interstitial emphysema that may further progress to the mediastinal space and subcutaneous emphysema: this phenomenon is known as Macklin effect [6]. SP was documented in $11 \%$ of 123 patients during the severe acute respiratory syndrome (SARS) epidemic in 2003, and the authors concluded that the presence of SP may be related to a poor outcome [7]. In our cohort of patients with COVID-19 pneumonia, the incidence of SP seems to be lower, around 6\%, possibly because COVID-19 pneumonia may have a different impact on the respiratory system from that of SARS.

SP is usually benign, as in a recent case that occurred during the COVID-19 epidemic in China [8]. The prognosis usually depends on the underlying conditions but it could represent a potential indicator of worsening disease. In our experience, the concomitant presentation of SP and diffuse COVID-19 pneumonia was associated with a severe clinical course characterized by sudden ARDS that required aggressive management and early intubation in three patients [9]. Furthermore, the presence of pneumomediastinum required some changes in the management of mechanical ventilation in order to minimize volutrauma and prevent its expansion. Avoidance of overventilation, adoptions of measures to limit hyperinflation and the cautious use of high levels of positive end-expiratory pressure was required but, despite these precautions, mechanical ventilation had the effect of increasing the size of the pneumomediastinum, as observed in two of our patients.

Table 1. Clinical characteristics, radiological data and in-hospital outcomes.

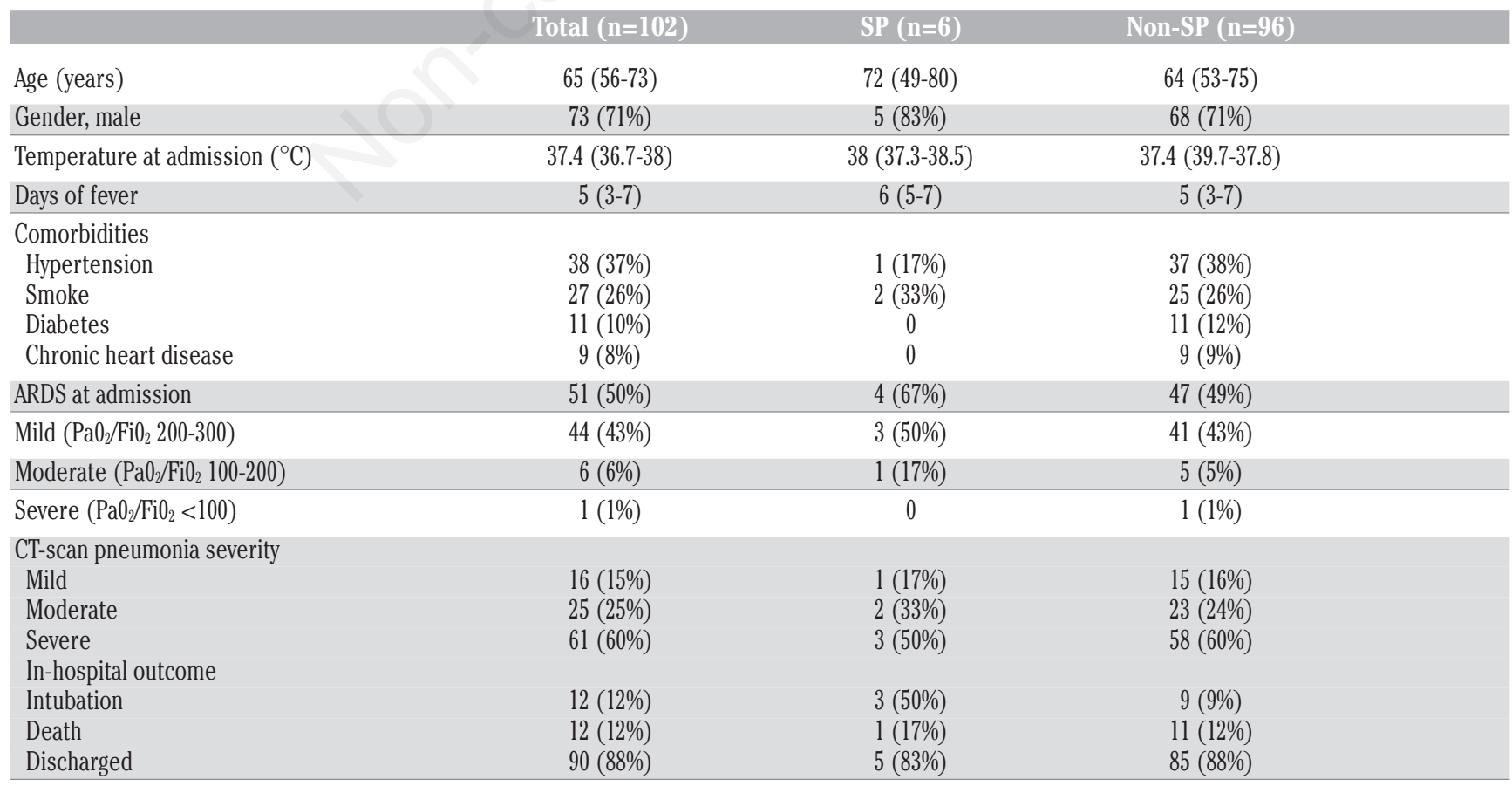

Data are median (IQR) or n (\%). SP, Spontaneous pneumomediastinum; ARDS, acute respiratory distress syndrome; $\mathrm{PaO}_{2}$, arterial oxygen partial pressure; FiO ${ }_{2}$, fraction of inspired oxygen; $\mathrm{CT}$, computer tomography. 


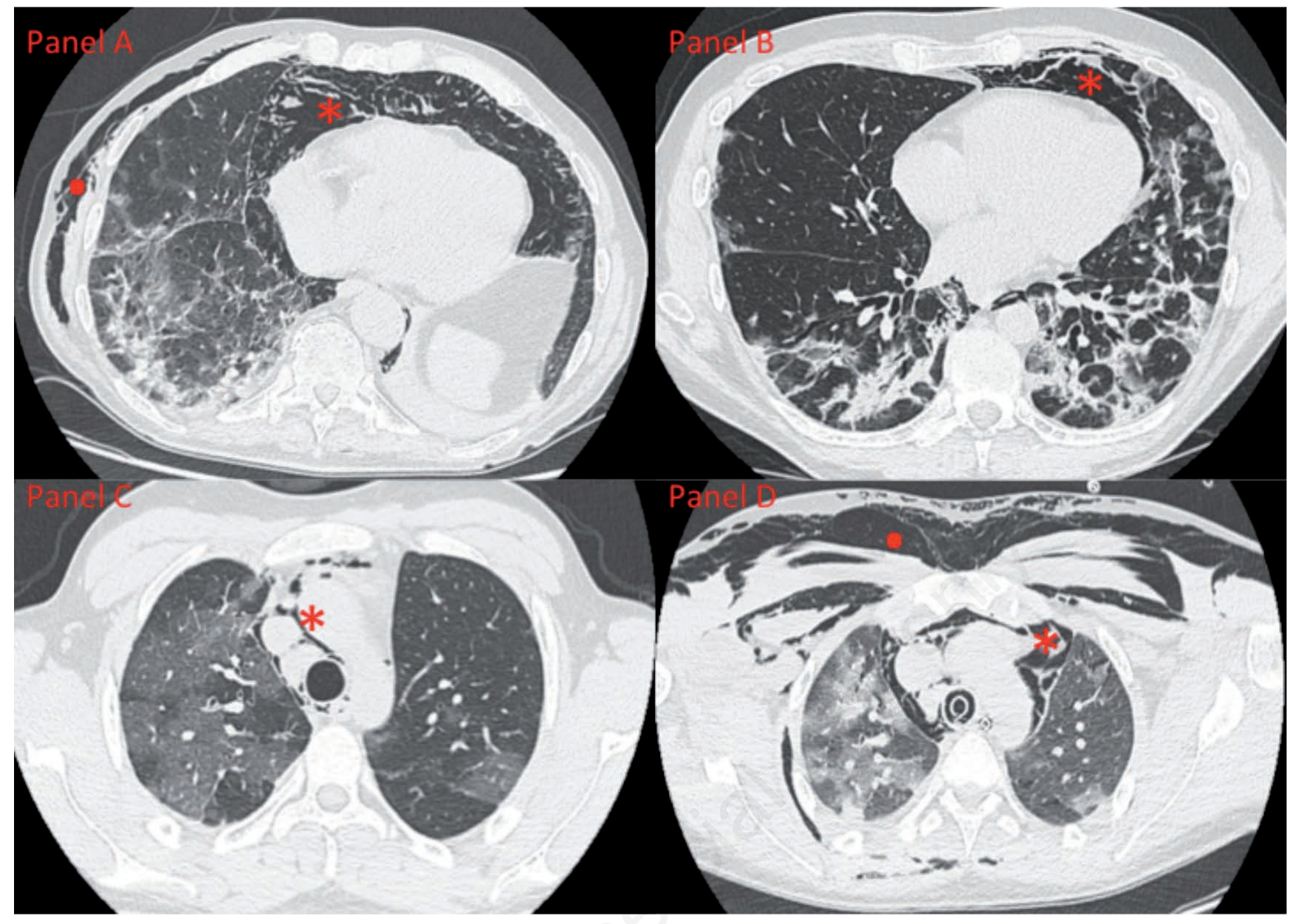

Figure 1. Axial CT-images. A) Peripheral airspace consolidations and ground glass densities in the right lower lobe, a large pneumomediastinum around the heart, and diffuse subcutaneous emphysema on the right side. B) Multiple patchy linear opacities with a perilobular pattern in both lungs, and a moderate amount of air in the anterior mediastinum. C) Extensive patchy ground glass density in the right upper lobe, less extensive similar densities in the left upper lobe, and a moderate pneumomediastinum. D) Chest CT-scan after two days of endotracheal intubation showing ground glass densities progressing to consolidation in the right upper lobe, diffuse subcutaneous emphysema, and more evident mediastinal emphysema than in the previous scan.

Table 2. Laboratory data at admission.

\begin{tabular}{lccc} 
& Normal range & SP $(\mathrm{n}=6)$ & Non-SP $(\mathbf{n}=96)$ \\
D-dimer $(\mathrm{mcg} / \mathrm{ml})$ & $0-0.5$ & $3(2.1-7.2)$ & $0.9(0.7-6.3)$ \\
Tn $(\mathrm{ng} / \mathrm{L})$ & $0-34$ & $10.5(5.1-134)$ & $9.2(4.7-25)$ \\
\hline CRP $(\mathrm{mg} / \mathrm{L})$ & $0-5$ & $109(38-263)$ & $50(16-117)$ \\
WBC $\left(* 10^{3} / \mathrm{mm}^{3}\right)$ & $3.9-10.6$ & $10560(4920-20255)$ & $5265(4692-8100)$ \\
\hline LC $(\%)$ & $20-45$ & $16(8-41)$ & $11(8-15)$ \\
Hb $(\mathrm{g} / \mathrm{dL})$ & $13.7-17.5$ & $14.4(12.2-15.4)$ & $14.1(13.1-15.1)$ \\
\hline PTL $\left(* 10^{3} / \mathrm{mm}_{3}\right)$ & $150-400$ & $156(127-200)$ & $183(139-252)$ \\
ALT $(\mathrm{U} / \mathrm{L})$ & $0-41$ & $37(19-92)$ & $18(38-44)$ \\
\hline AST $(\mathrm{U} / \mathrm{L}$ & $10-40$ & $60(32-91)$ & $36(26-59)$ \\
INR & $0.8-1.2$ & $1(0.9-1.2)$ & $1(0.7-1.1)$ \\
\hline LDH (U/L) & $<248$ & $236(167-305)$ & $367(235-487)$ \\
Creatinine $(\mathrm{mg} / \mathrm{dL})$ & $0.7-1.18$ & $0.87(0.7-1.2)$ & $0.96(0.8-1.2)$ \\
\hline
\end{tabular}

Data are median (IQR) or n (\%). SP, Spontaneous pneumomediastinum; Tn, troponin; CRP, C-reactive protein; WBC, white blood cell count; LC, lymphocyte count; PTL, platelets; ALT, alanine aminotransferase; AST, aspartate transaminase; INR, international normalized ratio; LDH, lactate dehydrogenase; $\mathrm{EGFR}$, estimated glomerular filtration rate. 


\section{Conclusions}

In conclusion, SP is a possible complication of severe COVID-19 pneumonia that can affect patient management and clinical outcomes.

\section{References}

1. Kouritas VK, Papagiannopoulos K, Lazaridis G, et al. Pneumomediastinum. J Thorac Dis 2015;7:S44-9. doi: 10.3978/j.issn.2072-1439.2015.01.11

2. Caceres M, Ali SZ, Braud R, et al. Spontaneous pneumomediastinum: a comparative study and review of the literature. Ann Thorac Surg 2008;86:962-6. doi: 10.1016/j.athoracsur.2008. 04.067

3. Remuzzi A, Remuzzi G. COVID-19 and Italy: what next? Lancet 2020;395:12251228. doi: 10.1016/S0140-6736(20) 30627-9

4. China National Health Commission. Chinese Clinical Guidance for COVID-19 Pneumonia Diagnosis and Treatment (7th edition). March 4, 2020. Accessed on: 10 April 2020. Available from: http://kjfy.meetingchina.org/msite/news/ show/cn/3337.html

5. WHO. Clinical management of severe acute respiratory infection when COVID-19 is suspected. Interim Guidance from WHO. March 13, 2020. Accessed on: 10 April 2020. Available from: https://www.who.int/publications-detail/clinical-management-of-severe-acute-respiratory-infection-when-novelcoronavirus-(ncov)-infection-is-suspected

6. Wintermark M, Schnyder P. The Macklin effect: a frequent etiology for pneumomediastinum in severe blunt chest trauma. Chest 2001;120:543-7. doi: 10.1378/chest.120.2.543

7. Chu CM, Leung YY, Hui JY, et al. Spontaneous pneumomediastinum in patients with severe acute respiratory syndrome. Eur Respir J 2004;23:802-4. doi: 10.1183/09031936.04. 00096404

8. Zhou C, Gao C, Xie Y, Xu M. COVID-19 with spontaneous pneumomediastinum. Lancet Infect Dis 2020;20:510. doi: 10.1016/S1473-3099(20)30156-0

9. Sahni S, Verma S, Grullon J, Esquire A, et al. Spontaneous pneumomediastinum: time for consensus. N Am J Med Sci 2013;5:460-4. doi:10.4103/1947-2714.117296 